Background Stigma and discrimination related to HIV and populations at high risk of HIV have the potential to impede the implementation of effective HIV prevention and treatment programmes. We will conduct an implementation science study of HIV-related stigma in communities and health settings within a large, pragmatic cluster-randomised trial of a universal testing and treatment intervention for HIV prevention in Zambia and South Africa and assess how stigma affects, and is affected by, implementation of this intervention.

Methods A mixed-method evaluation will be nested within HPTN071/PopART (Clinical Trials registration number NCT01900977), a three-arm trial comparing universal door-todoor delivery of HIV testing and referral to prevention and treatment services, accompanied by either an immediate offer of antiretroviral treatment to people living with HIV (PLHIV) regardless of clinical status, or an offer of treatment in-line with national guidelines, with a standard-of-care control arm. The primary outcome of HPTN071/PopART is HIV incidence measured among a cohort of 52,500 individuals in 21 study clusters. Our evaluation will include integrated quantitative and qualitative data collection and analysis in all sites. We will collect quantitative data on indicators of HIV-related stigma over three years from large probability samples of community members, health workers, and PLHIV, and qualitative data, including in-depth interviews and observations from members of these same groups sampled purposively. In analysis we will: (i) compare HIV-related stigma measures between study arms, (ii) link data on stigma to measures of the success of implementation of the intervention, (iii) explore changes in the drivers and manifestations of stigma in study communities and the health system.

Discussion Using a novel study-design nested within a large, pragmatic trial we will evaluate the extent to which HIV-related stigma affects and is affected by the implementation of a comprehensive combination HIV prevention intervention including a universal test and treatment approach.

Disclosure of interest statement HPTN 071 is sponsored by the National Institute of Allergy and Infectious Diseases (NIAID) under Cooperative Agreements UM1-AI068619, UM1AI068617, and UM1-AI068613, with funding from the US President's Emergency Plan for AIDS Relief (PEPFAR). Additional funding is provided by the International Initiative for Impact Evaluation (3ie) with support from the Bill and Melinda Gates Foundation, as well as by NIAID, the National Institute on Drug Abuse (NIDA) and the National Institute of Mental Health (NIMH), all part of NIH. The content is solely the responsibility of the authors and does not necessarily represent the official views of the NIAID, NIMH, NIDA, PEPFAR, 3ie, or the Bill and Melinda Gates Foundation. We have no conflicts of interest to declare.

\section{P14.14 INTERSECTING STIGMAS: A FRAMEWORK FOR DATA COLLECTION AND ANALYSIS OF STIGMAS FACED BY PEOPLE LIVING WITH HIV AND KEY POPULATIONS}

${ }^{1} \mathrm{JR}$ Hargreaves*, ${ }^{2} \mathrm{~A}$ Stangl, ${ }^{1,3} \mathrm{~V}$ Bond, ${ }^{4} \mathrm{G}$ Hoddinott, ${ }^{1} \mathrm{~S}$ Krishnaratne, ${ }^{4} \mathrm{H}$ Mathema ${ }^{3} \mathrm{M}$ Moyo, on behalf of the HPTN071 (PopART) Study Team. 'London School of Hygiene and Tropical Medicine; ${ }^{2}$ International Center for Research on Women; ${ }^{3}$ ZAMBART Project; ${ }^{4}$ Desmond Tutu Tuberculosis Centre, Stellenbosch University

\subsection{6/sextrans-2015-052270.526}

Introduction Stigma can impede the implementation of HIV prevention and treatment programmes. Many measurement scales have been developed, though few have addressed intersecting stigmas: that is to say, multiple stigmas faced by people living with HIV, and key populations at high risk of HIV.

Methods We developed an approach to measure intersecting stigmas nested within the HPTN 071 (PopART) trial, conducted in South Africa and Zambia. We adopted best-practice wording to assess key domains of stigma, building on a process of global consultative indicator harmonisation. We designed nested data collection items in surveys for parallel use among the general population, health workers and people living with HIV (PLHIV) - including health workers living with HIV - to assess the same phenomena from multiple perspectives. We also designed "parallel" assessments of key population stigma within the health care worker survey to compare the attitudes and perceptions of health care workers towards PLHIV, and those at increased risk of HIV infection, such as migrants, sex workers, MSM, young women, and people with disabilities.

A core set of seven questions was included in the HPTN071 (PopART) surveys conducted among the general population (over 10,000 individuals), PLHIV (about 4000 individuals) and health care workers (over 1000 individuals). These items assessed two key domains of HIV stigma: drivers of stigma and manifestations of stigma. In the health care worker survey, questions specific to key populations were also included, to assess health care worker attitudes, experiences and perceptions about those at increased risk of HIV as listed above. We developed an approach to visually represent the complex data set using methods based on social-network analysis.

We developed an approach to assess intersecting HIV-related stigmas, incorporating data collected from different population groups. Data collection is ongoing.

Disclosure of interest statement HPTN 071 is sponsored by the National Institute of Allergy and Infectious Diseases (NIAID) under Cooperative Agreements UM1-AI068619, UM1AI068617, and UM1-AI068613, with funding from the US President's Emergency Plan for AIDS Relief (PEPFAR). Additional funding is provided by the International Initiative for Impact Evaluation (3ie) with support from the Bill and Melinda Gates Foundation, as well as by NIAID, the National Institute on Drug Abuse (NIDA) and the National Institute of Mental Health (NIMH), all part of NIH. The content is solely the responsibility of the authors and does not necessarily represent the official views of the NIAID, NIMH, NIDA, PEPFAR, 3ie, or the Bill and Melinda Gates Foundation. We have no conflicts of interest to declare.

\section{P14.15 IN THE SYSTEM (TONGAN SYSTEM) BUT OUT OF PLACE}

Joey Joleen Siosaia*. WCA Mataele - Tonga Leitis' Association/Pacific Sexual Diversity Network

\subsection{6/sextrans-2015-052270.527}

Introduction Since HIV/AIDS was discovered in the early 80 s, and the first AIDS Victim came to Tonga from the United States, 19 people have lost their lives to the virus leaving many children and MSM/TG with more discriminations for our Communities. Since then there has been a lot of Stigma and Discriminations on our young MSM/TGs. These children have been forced out of school, engaged in hard labour, prostitution or high risk behaviours that make them vulnerable to contracting HIV. With support from international donors and local resource efforts.

Methods Tonga Leiti's Association (TLA) has a long and complex history. It's a history of survival. Its history has been 
governed with diverse and contesting ways of understanding, filled with contradictions, categorization, labelling and marginalisation. Hence, these ways of knowing problematizes TLA and its pupils in a normative ways. It situates them outside the 'normal' 'Anga FakaTonga' (Tongan ways) mainly because their distinctive behaviours are perceived as falling outside the 'Anga FakaTonga' awareness of acceptable behaviour and attitude. It is, therefore, position Leitis as 'children of the devil'. The TLA and its pupils are variously described as being at-risk, vulnerable, stigmatised and lacking agency.

Results Moreover, their lives are equated with having a 'lack of hope and mobility' so they are seen as being on a pathway that leads to 'poverty' and isolation from normative model and space in Tongan society. We recognised that through positioning outside the 'normal Anga FakaTonga', there is a tendency to perceive TLA and its pupils in a simplistic way and thus treat them as a marginal community.

Conclusion However, it must be acknowledged that this tendency to stereotype TLA and its pupils displays a lack of awareness for their:

- active agency and the fact they have some control over their life journey,

- contributions to Tongan civil society,

- distinctive cultural, gifts and artistic talents,

- support to the business communities,

- capacity to support the younger Leiti's pupils,

- capacity to educate the general public regarding the sexually transmitted diseases - HIV/AIDS and STI's, and

- Marketing of Tonga to the international communities.

Disclosure of interest statement The Tonga Leiti's Association (TLA) is a Registered Association in the Kingdom of Tonga. The TLA works as an advocacy organisation focused on the promotion of the rights and creating a dialogue about issues facing the transgendered community in Tonga. The TLA aims to oversee any opportunities addressing human rights and also to carry out awareness to its members. As a Registered Association of Tonga the TLA is required to have board members which include representatives from the government and non-government organisations. The TLA is a voluntary organisation and all of the staff are volunteers. I am the current Executive Director of the TLA and the current Chairperson of the Pacific Sexual Diversity Network. The Hon. Lupepau'u Tuita from the Tongan Royal Family is the Patron of the TLA. At the Annual General Meeting there are appointed positions that are tasked with the administration and operating of the Association.

\section{P14.16 SELF REPORTED HIV DISCRIMINATION AND WILLINGNESS TO DISCLOSE HIV SEROPOSITIVITY AMONG A SAMPLE OF TURKS AND CAICOS ISLANDERS}

${ }^{1}$ A Robinson*, ${ }^{2}$ SD Malcolm. 'National AIDS Program, Ministry of Health, Grand Turk, Turks and Caicos; ${ }^{2}$ National Epidemiology and Research Unit, Ministry of Health, Grand Turk, Turks and Caicos

\subsection{6/sextrans-2015-052270.528}

Background Human Immunodeficiency Syndrome (HIV) is a major source of concern in the Turks and Caicos Islands (TCI), a small archipelago of islands in the Caribbean region. Since transmission is predominantly through sexual contact, it is important to understand the dynamics of sexual relationships in this country, including the likelihood of HIV seropositivity disclosure. Knowledge about one's partner's seropositivity can guide both the sexual behaviour and HIV testing decision-making processes. The objective of the study was thus to examine factors associated with willingness to disclose HIV status in a general TCI sample, including self-reported discrimination towards HIV positive persons, which has rarely been examined in relation to disclosure.

Methods The current study is based on secondary analysis of the 2011 Knowledge, Attitudes Practices and Behaviours survey data $(\mathrm{N}=837 ; 55 \%$ female; mean age $=31.28(\mathrm{STD}=9.3))$. Multivariate logistic regression examined the association between willingness to disclose HIV seropositivity to partner (Yes/No) and gender, age, sexual partner number, church attendance, cohabitation status, HIV treatment knowledge, ever tested status and self-reported HIV discrimination.

Results Nearly half of the participants were unwilling to disclose HIV seropositivity (44.8\%). Moreover, willingness to disclose HIV seropositivity was related to having one or less sexual partner $(\mathrm{OR}=1.98,95 \% \mathrm{CI}=1.29-3.01)$, non-regular church attendance $(\mathrm{OR}=1.55,95 \% \mathrm{CI}=1.10-2.19)$, cohabitation $(\mathrm{OR}=0.67,95 \% \mathrm{CI}=0.47-0.97)$, knowing about HIV treatment $(\mathrm{OR}=0.50,95 \% \mathrm{CI}=0.32-0.80)$ and having an HIV test $(\mathrm{OR}=0.43,95 \% \mathrm{CI}=0.26-0.63)$. Self-reported discrimination was also related to willingness to disclose HIV seropositivity $(\mathrm{OR}=0.62,95 \% \mathrm{CI}=0.44-0.88)$.

Conclusion Several factors were associated with willingness to disclose HIV seropositivity, including self-reported discrimination. These findings may have implications on post HIV test counselling initiatives, which should include a focus on education.

Disclosure of interest statement Nothing to declare.

\section{P14.17 SURVEILLANCE FOR SEXUALLY TRANSMITTED INFECTIONS AMONG FEMALE SEX WORKERS IN INNER- CITY JOHANNESBURG}

${ }^{1}$ V Black, ${ }^{2}$ V Maseko, ${ }^{1} F W$ Venter, ${ }^{2} F$ Radebe, ${ }^{1} S$ Mullick, ${ }^{1} H V$ Rees, ${ }^{2,3}$ DA Lewis. ${ }^{1}$ Wits Reproductive Health and HIV Institute, Faculty of Health Sciences, University of Witwatersrand; ${ }^{2}$ Centre for HIV and STIS, National Institute for Communicable Diseases (NHLS); ${ }^{3}$ Western Sydney Sexual Health Centre and University of Sydney, Sydney, Australia

\subsection{6/sextrans-2015-052270.529}

Introduction Sexually transmitted infection (STI) surveillance informs empiric antibiotic recommendations for syndromic management. Asymptomatic STIs are an important source of new infections which increase the risk of HIV transmission and acquisition. As female sex-workers (FSW) are key HIV/STI transmitters, microbiological studies within this population provide critical HIV/STI strategic information.

Methods Female sex workers (FSW) attending a sex-worker clinic or outreach brothel services, between March and December 2014 in inner-city Johannesburg were enrolled. A questionnaire and clinical examination were completed. Specimens were collected from the throat, urethra, cervix, vagina and rectum. Laboratory investigations included (i) culture for Neisseria gonorrhoeae (NG) with susceptibility testing for cefixime and ceftriaxone, (ii) DNA amplification using a multiplex PCR for NG, C. trachomatis (CT), M. genitalium (MG) and T. vaginalis (TV), (iii) microscopic examination for bacterial vaginosis (BV), and (iv) serological testing for syphilis, hepatitis B and HIV. Participants with STIs were treated either at the initial visit (symptomatics) or following recall (asymptomatics).

Results The mean age of the 268 enrolled FSW was 31.5(IQR $27-35$ ) and mean duration of sex work was 4.9 years (range 2 months-30 years). Condom use was consistent among 88 